\title{
¿Es posible hacer divertido y ameno el estudio de la Bioquímica?: La gamificación para aprender
}

\section{Is it possible to make the study of Biochemistry fun and enjoyable? The gamification to learn}

\author{
Ángel Llamas Azúa, Manuel Tejada Jiménez, David González Ballester, Emilio \\ Fernández Reyes
}

Fecha de recepción: 23/09/2018; Fecha de revisión: 25/02/2019; Fecha de aceptación: 20/05/2019

Cómo citar este artículo:

Llamas, A., Tejada, M., González, D. y Fernández, E. (2019). ¿Es posible hacer divertido y ameno el estudio de la bioquímica?: La gamificación para aprender. Revista de Innovación y Buenas Prácticas Docentes, 8(2), pp.1-11.

Autor de Correspondencia: Ángel Llamas, allamas@uco.es

Departamento de Bioquímica y Biología Molecular. Campus de Rabanales, Edif C-6, plata baja. 14071 Córdoba.

\section{Resumen:}

La Gamificación es el empleo de mecánicas de juego con el fin de potenciar la motivación, la concentración, el esfuerzo y otros valores positivos comunes a todos los juegos. Se trata de una nueva y poderosa estrategia para influir y motivar a grupos de estudiantes. Memrise es una herramienta gratuita de aprendizaje online con cursos creados por su comunidad. Memrise permite aumentar la rapidez y la facilidad del aprendizaje, realizándose además de una forma amena. También, fomenta la competitividad entre los estudiantes a través de un sistema de puntuación. Los cursos Memrise son también de autoaprendizaje, a través de las Mems, que son frases cortas o bien imágenes que los estudiantes adjuntan de forma individual a cada una de las preguntas. Gracias a este proyecto se ha creado un curso Memrise de 1081 preguntas que puede ser utilizado para cualquier asignatura de bioquímica fundamental. Este curso se ha empleado en la asignatura bioquímica de primero de Enfermería y se ha analizado la utilización por parte del alumnado. Llegándose a la conclusión que su utilización ha sido muy notable por parte del alumnado y que ha repercutido positivamente en la preparación de los exámenes por parte del estudiante.

Palabras clave: Gamificación, motivación, Memrise, autoaprendizaje.

Abstract:

Gamification is the use of game mechanics in order to enhance motivation, concentration, effort and other positive values common to all games. It is a new and powerful strategy to influence and motivate groups of students. Memrise is a free online learning tool with courses created by the community. Memrise allows you to increase the speed and ease of learning, done in an enjoyable way. Also, it encourages competitiveness among students through a scoring system. The Memrise courses are also self-learning, through the Mems, which are short sentences or images that students attach individually to each of the questions. Thanks to this project, a Memrise course has been created for any subject of fundamental Biochemistry. This course consists of 1081 questions for the subject Biochemistry of Nursing and has analyzed the use by students. Arriving at the conclusion that its use has been very remarkable on the part of the students and that it has had a positive impact on the student's preparation for the exams 
Llamas, A. Tejada-Jiménez, M. González-Ballester, D. Fernández, E.

Key Words: Gamification, motivation, Memrise, self-learning. 


\section{INTRODUCCIÓN}

Este proyecto pretende ofrecer al alumno una serie de actividades realizadas de manera virtual para el desarrollo de sus competencias en el campo de la bioquímica. Este proyecto será aplicable a cualquier asignatura básica de bioquímica. Diferentes asignaturas básicas de bioquímica son obligatorias en una gran cantidad de distintos grados de esta universidad, por citar algunos de ellos, en los grados de Enfermería, Fisioterapia, Medicina, Biología, Química, bioquímica y Ciencias y tecnología de los alimentos. Lo que indica que, éste es un proyecto multidisciplinar al integrar asignaturas de titulaciones muy diferentes.

El Espacio Europeo de Educación Superior perfila un modelo de aprendizaje centrado en el alumno, en el que el profesor es un facilitador del aprendizaje, el alumno tiene una autonomía creciente y las Tecnologías de la Información y la Comunicación (TICs) desempeñan un papel relevante, por cuanto que la enseñanza presencial abre cada vez más paso a la virtual, y a la investigación del alumno y la construcción de su propio aprendizaje. La extensión de internet/wifi a todos los ámbitos de la sociedad ha permitido que la universidad pueda llegar directamente al hogar del alumno. Este es el concepto generalmente conocido por el término "elearning". El uso de las actividades e-learning, es un proceso de aprendizaje en el que los materiales de formación se entregan a los usuarios a través de internet. Estas actividades formativas facilitan la comunicación entre el profesor y los alumnos según determinadas herramientas sincrónicas y asincrónicas de la comunicación.

El Coordinador de este proyecto tiene 7 años de experiencia como coordinador en la docencia de la asignatura bioquímica de $1^{\circ}$ del Grado de Enfermería. Por lo que en un principio, de concederse este proyecto, se le aplicará a este curso. El porcentaje de alumnos al que va dirigido este proyecto es el 100\% del grado (120 alumnos en 1은 de enfermería), ya que en la mayoría de ellos bioquímica es una asignatura obligatoria. Además, debido al temario bastante similar de la docencia en bioquímica en el resto de grados (como lo atestigua la lectura de las distintas guías docentes) este proyecto será plenamente exportable a los distintos grados en los que se imparte bioquímica. La asignatura bioquímica de 1ำ de Enfermería consta de 6 créditos ECTS. Con 3 horas por semana de gran grupo (GG) y 14 sesiones de Grupo Mediano (GM) que se realizan en sesiones de 1 hora durante 14 semanas. En la actualidad la bioquímica de $1^{\circ}$ de Enfermería consta de 15 temas de teoría que generalmente se imparten en las sesiones de GG. En este tipo de asignaturas con solo 6 créditos ECTS es difícil para los profesores llevar un control de los avances de los estudiantes en la asimilación de los conocimientos teóricos y prácticos.

Creemos que tenemos que avanzar aún más mediante el empleo de la nueva metodología denominada gamificación (gamification en el ámbito anglosajón). La gamificación es el empleo de mecánicas de juego en entornos y aplicaciones no lúdicas con el fin de potenciar la motivación, la concentración, el esfuerzo, y otros valores positivos comunes a todos los juegos, en el ámbito de la enseñanza de la bioquímica. La originalidad de este proyecto radica en aplicar la gamificación, como una nueva y poderosa estrategia para influir y motivar a los estudiantes en el ámbito de la bioquímica. 
Moodle es una excelente herramienta que nos brinda la UCO a los profesores para la realización de un gran número de actividades, ya fundamentales e imprescindibles, para cualquier asignatura. Sin embargo, algunos profesores hemos detectado que en particular los cuestionarios realizados a través del Moodle adolecen de una serie de limitaciones que podrían ser mejoradas. Los cuestionarios a través de Moodle son adecuados para calificar los conocimientos, pero no tanto para facilitar el auto-aprendizaje por los estudiantes. Por ejemplo, Moodle no permiten que los estudiantes adapten las preguntas a sus conocimientos, al no poder ellos editar las preguntas e introducirles ayudas para que les resulte más fácil su asimilación. Moodle no permite la competición entre los distintos estudiantes de curso. Moodle no tiene aplicaciones (Apps) para la tableta o el móvil. Estas y otras limitaciones del Moodle podrían ser subsanadas mediante la utilización de la plataforma Memrise (http://www.memrise.com/home/).

Memrise es una herramienta online gratuita de gamificación del aprendizaje con cursos creados por la comunidad. Sus cursos son principalmente usados para enseñar idiomas, pero también son utilizados para enseñar otras materias académicas y no académicas. Memrise usa flashcards aumentadas con reglas mnemotécnicas, parcialmente reunidas a través del pensamiento masivo, que permite aumentar la rapidez y la facilidad del aprendizaje, realizándose además de una forma más amena.

Los cursos Memrise, contrariamente a lo que permite Moodle, son además muy dinámicos, en el sentido que una vez que se completa el cuestionario de uno de los temas, cada cierto tiempo se reactivan y el programa solicita al estudiante que los vuelva a hacer para que no decaiga la memoria de lo aprendido. A medida que se van repitiendo los cuestionarios, el tiempo que pasa entre cada uno de los recordatorios va aumentando progresivamente.

Los cursos Memrise son además de autoaprendizaje, en el sentido que, Memrise hace que cada estudiante tenga la posibilidad de introducir, en cada una de las preguntas que constituyen los cuestionarios del curso, los llamados Mems. Los Mems son frases cortas, palabras o bien imágenes que los estudiantes adjuntan de forma individual a cada una de las preguntas y que les ayuda a la asimilación y memorización de la respuesta. Estos Mems una vez creados se comparten por toda la comunidad, y cada estudiante decide si coger algunos de los ya creados o crear los suyos propios. Es decir la filosofía es distinta, los cuestionarios Memrise facilitan y motivan el aprendizaje y los del Moodle están más bien destinados a calificar el aprendizaje.

Además, Memrise fomenta la competitividad entre los estudiantes a través de un sistema de puntuación. Los estudiantes a medida que van realizando el curso van obteniendo una puntuación en función de lo bien que respondan a las preguntas de los cuestionarios. Estas puntuaciones son visibles por los demás estudiantes inscritos en el curso y por los profesores. Otra diferencia significativa entre los cuestionarios realizados a través del Moodle y los de Memrise es que esta última plataforma tiene versiones para el móvil y tabletas, de lo cual adolece actualmente el Moodle de la UCO. Esto hace que los estudiantes tengan continuamente disponible y de forma fácil la posibilidad de dedicar tiempo al estudio de los cursos hechos en la plataforma Memrise, como sería el caso del de bioquímica que se pretende crear con este proyecto de innovación docente. Los cursos en Memrise también poseen un foro, donde los estudiantes se comunican entre sí y con los profesores. 


\section{DESARROLLO DE LA EXPERIENCIA DE INNOVACIÓN}

Uno de los objetivos es diseñar actividades motivadoras, atractivas y resueltas, relacionadas con la enseñanza universitaria de la bioquímica. También, promover un aprendizaje virtual activo. Utilizar dichas actividades para la autoevaluación del alumno, para que el alumno estudie cada unidad temática y para ser usadas por el profesor en la evaluación de la asignatura.

Utilizando la bibliografía (Berg et al., 2013; Devlin, 2004; Luque y Herráez 2001; Nelson y Cox, 2014; Tymoczko et al., 2014; Voet et al., 2014) como base para la creación de las preguntas, se han creado un total de 1081 preguntas. Lo que equivale a una media de 72 preguntas para los 15 temas de los que consta la asignatura. Utilizando las herramientas online de la plataforma Memrise se han introducido cada una de ellas en un curso de nueva creación, llamado "Bioquímica Enfermería". Una vez completada la creación del curso en Memrise, se ha puesto disponible para la comunidad $y$ se ha hecho saber a los estudiantes de su existencia, de su funcionamiento y de la importancia de su realización para su avance en la asimilación de los conocimientos de la asignatura de bioquímica. Se ha pretendido con este curso que el estudiante disponga de una forma dinámica, amena, divertida, competitiva y más accesible que los típicos cuestionarios a través del Moodle, de mejorar sus conocimientos de la asignatura bioquímica. También, a través de la herramienta de los Mems se pretende que los estudiantes sean partícipes de su propio aprendizaje y lo puedan adoptar a su lógica de pensamiento.

Las competencias que se han potenciado durante la realización de este proyecto son las relacionadas con la asimilación de los conocimientos teóricos y el uso de las TIC, entre ellas:

CB1: Que los estudiantes hayan demostrado poseer y comprender conocimientos en un área de estudio que parte de la base de la educación secundaria general, y se suele encontrar a un nivel que, si bien se apoya en libros de texto avanzado, incluye también algunos aspectos que implican conocimientos procedentes de la vanguardia de su campo de estudio.

CB3: Que los estudiantes tengan la capacidad de reunir e interpretar datos relevantes (normalmente dentro de su área de estudio) para emitir juicios que incluyan una reflexión sobre temas relevantes de índole social, científica o ética.

CB5: Que los estudiantes hayan desarrollado aquellas habilidades de aprendizaje necesarias para emprender estudios posteriores con un alto grado de autonomía.

CU2: Conocer y perfeccionar el nivel de usuario en el ámbito de las TICs.

CET3: Conocer y aplicar los fundamentos y principios teóricos y metodológicos.

CEM1: Conocer e identificar la estructura y función del cuerpo humano. Comprender las bases moleculares y fisiológicas de las células y los tejidos. 
Dirección web de la plataforma donde se han creado y están disponibles las preguntas 1081 preguntas: http://www.memrise.com/home/. Los diferentes libros utilizados para la creación de las preguntas se pueden consultar en la sección de bibliografía.

\section{RESULTADOS}

Se pretende que el empleo de la gamificación a través de la utilización de los cursos Memrise como herramienta, fomenten la competitividad entre los estudiantes mediante un sistema de puntuaciones online. Memrise incita a la constancia en el estudio de los distintos temas mediante recordatorios. También los cursos Memrise propician el autoaprendizaje al dar la posibilidad de introducir en cada pregunta Mems que les sirva de ayuda a los estudiantes a la comprensión y asimilación de cada pregunta. El principal resultado que se espera obtener es que los estudiantes progresen de una forma continua en el estudio de la bioquímica de $1^{\circ}$ de Enfermería. Al disponer la asignatura de bioquímica de 1ํ de Enfermería de solo 3 horas de sesión de GG por semana, a algunos estudiantes les cuesta mantener la motivación y la constancia en su estudio durante el cuatrimestre. Los cursos Memrise facilitan el control por parte de los profesores del tiempo y avances realizados por cada uno de los estudiantes. En definitiva todo esto pretende que repercuta en un incremento de la nota media de la asignatura.

Se ha creado un curso de bioquímica en Enfermería. Que consta de 15 temas y un total de 1081 preguntas divididas entre los 15 temas e la asignatura. Cualquiera puede tener acceso libre a la aplicación y hacer las preguntas a través del enlace: https://www.memrise.com/course/540699/bioquimica-enfermeria/. Previamente dándose de alta con un correo electrónico, un nombre de usuario y palabra clave. Cuando los alumnos entran en Memrise en la sección de ciencias de la salud se encuentran con la página principal mostrada en la (figura 1). 


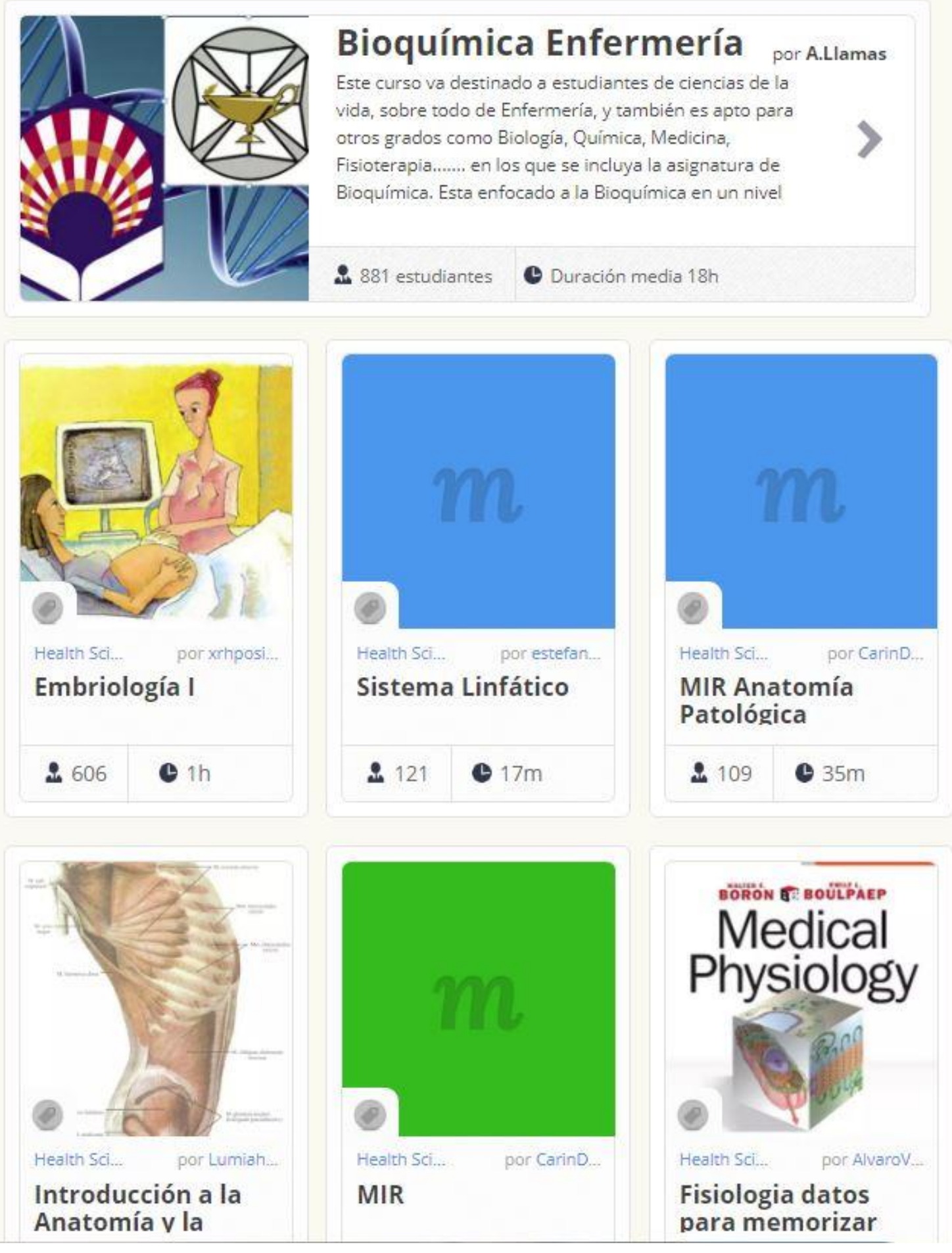

Figura 1. Página principal de los cursos de ciencia de la salud de Memrise.

Como se puede apreciar en la figura 1, actualmente Bioquímica de Enfermería es el curso que ha sido realizado por más personas dentro de la sección de ciencias de la salud, un total de 881 . El número tan elevado de usuarios realizando el curso, cuando los matriculados este año en la bioquímica de Enfermería son 
aproximadamente 120, se debe a que al curso tiene libre acceso cualquier usuario de la plataforma Memrise. Lo que es un indicador de la amplia aceptación que ha tenido por el resto de la comunidad. Como se puede observar en la misma figura el tiempo media de realización del curso completo son 18 horas.

A continuación, cuando el alumno entra en la citada aplicación informática (Fig. 2) puede empezar a hacer las correspondientes preguntas de cada uno de los temas que se vayan realizando en clase. A continuación, se muestra unos ejemplos de algunas de las 1081 preguntas disponibles en la aplicación creada para este proyecto de innovación docente (figura 2).

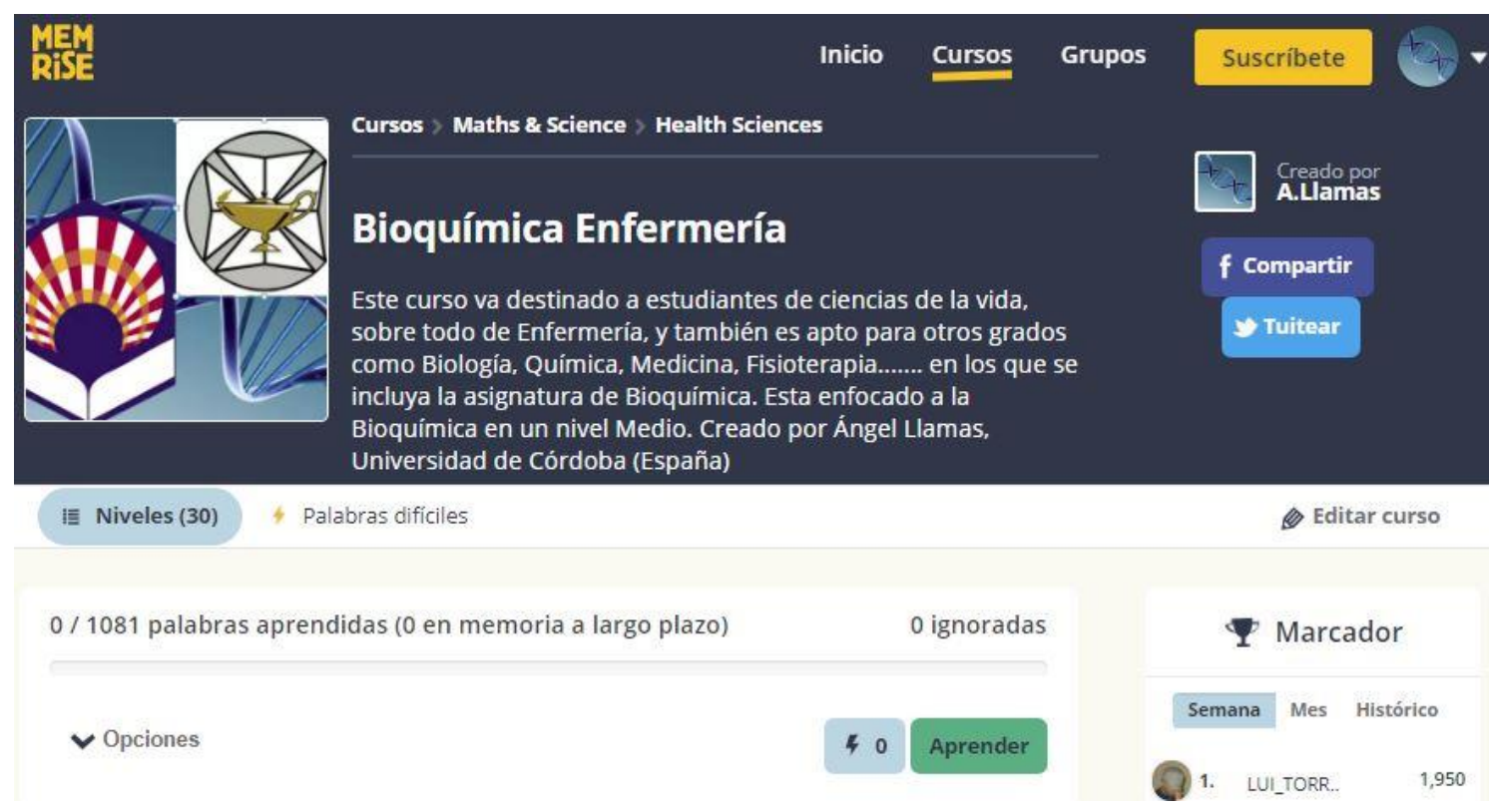

Figura 2. Página principal del curso creado en la aplicación Memrise.

Para aquellos conceptos que les resulten a los estudiantes difíciles de asimilar pueden crear recordatorios llamados Mems como el que ilustra la figura 3. 


\section{Los monosacáridos se clasifican atendiendo a su grupo funcional en}

Escoge la opción correcta

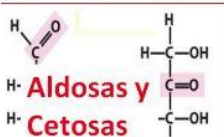

\section{Asimétrico}

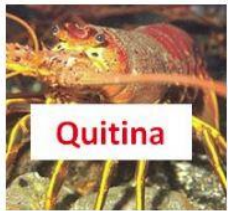

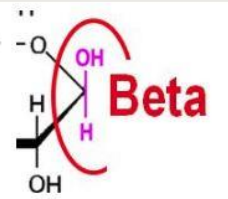

\section{Azúcar característico del ARN}

Escoge la opción correcta<smiles>[Y9]C1O[C@H](O)[C@@H](O)[C@H]1O</smiles>

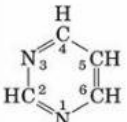

Pirimidinas

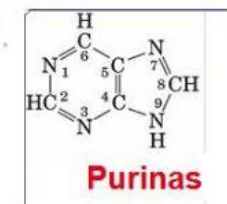

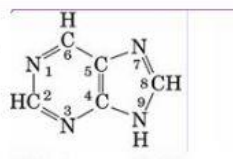

\section{Todas las células tienen una serie de ........ bioquímicas} similares

Escoge la opción correcta
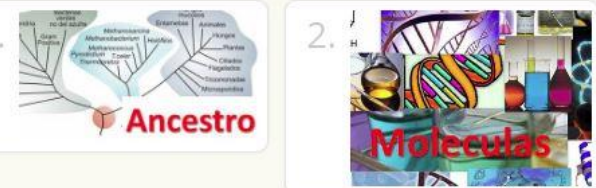

\section{Nucleotido}

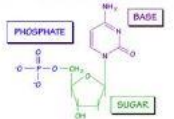

\section{Conjunto de reacciones}

(7) químicas que se realizan en el interior de la célula

Escoge la opción correcta

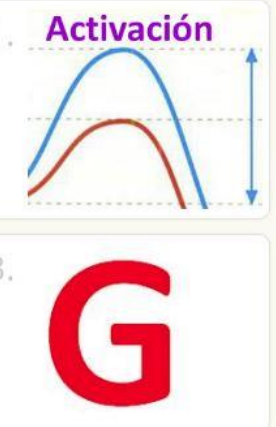

\section{Acopladas}

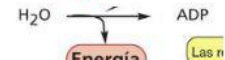
Energía Las $n$ , $+\mathrm{NH}_{4}+\stackrel{\longrightarrow}{\longrightarrow}$ glutam

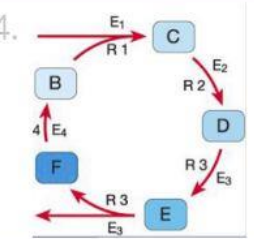

Figura 3. Ejemplo de 4 preguntas al azar de entre las 1081 preguntas en la aplicación Memrise de bioquímica para primero de Enfermería. 


\section{Proteínas}

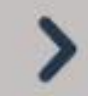

Siguiente

\section{Las secuencias de ADN codifican para}

Menos

Has elegido este mem:

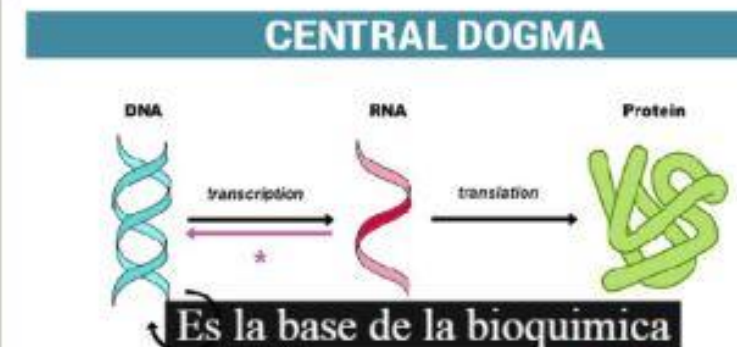

Figura 4. Ejemplo de un Mems creado por un estudiante para recordar la función del ADN.

Los profesores han podido comprobar cómo iba siendo el avance en la realización de las preguntas a lo largo de todo el curso. Mediante la comprobación del estado de las puntuaciones de cada alumno (Figura 5).

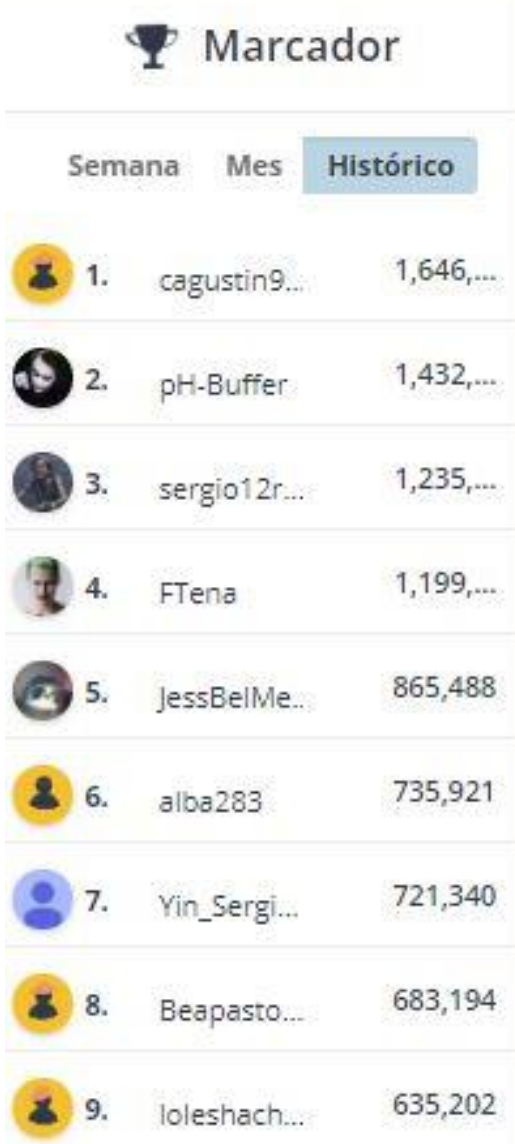

Figura 5. Estado de las puntuaciones obtenidas a medida que se va realizando el curso. 


\section{CONCLUSIONES}

Este nuevo curso de bioquímica general diseñado en Memrise ha mejorado el autoaprendizaje del estudiante al poder realizar de una manera amena e interactiva una de las tareas más arduas en bioquímica, la memorización de determinados conceptos difíciles de asimilar. También al dar la posibilidad de introducir en cada pregunta Mems que les sirva de ayuda a los estudiantes a la comprensión y asimilación de cada pregunta, los mismos estudiantes han participado en su aprendizaje. Este curso creado ha facilitado el control por parte de los profesores del tiempo y avances realizados por cada uno de los estudiantes. En definitiva, con todo esto, se ha conseguido que repercuta positivamente en un incremento de la nota media de la asignatura de bioquímica de primero de Enfermaría. También es de destacar que los alumnos han llegado al examen con más confianza ya que han practicado uno de los tipos de preguntas que se les hace en los exámenes de esta asignatura. La realización de los cuestionarios les ha ayudado a los estudiantes a saber el grado de asimilación de los conocimientos de los distintos temas de teoría.

\section{Agradecimientos}

Este trabajo ha sido posible gracias a: MINECO (Ministerio de Economía y Competitividad, Spain, Grant no. BFU2015-70649-P, Junta de Andalucía (P12-BIO502), European FEDER program, "Plan Propio de la Universidad de Córdoba", y U.E.INTERREG [0055_ALGARED_PLUS_5_E].

\section{REFERENCIAS}

Berg, J.M., Tymoczko, J.L. y Stryer L. (2013). Bioquímica (7a Ed.). Barcelona: Reverté.

Devlin, T.M. (2004). Bioquímica, Libro de Texto con Aplicaciones Clínicas (4⿳亠口冋 Ed), Barcelona: Editorial Reverté.

Luque, J. y Herráez, A. (2001). Texto Ilustrado de Biología Molecular e Ingeniería Genética. Concepto, Técnicas y aplicaciones en Ciencias de la Salud. Ed. Harcourt.

Nelson, D.L, Cox, M.M. (2014). Lehninger Principios de Bioquimica, Barcelona: Ediciones Omega.

Tymoczko, J.L., Berg, J.M., Stryer, L. (2014). Bioquímica: Curso básico (2a Ed.). Barcelona: Editorial Reverté.

Voet, D., Voet, J.G. y Pratt, C.W. (2007). Fundamentos de Bioquímica. La vida a nivel molecular (2 $\left.{ }^{\mathrm{a}} \mathrm{Ed}\right)$. Madrid: Editorial Médica Panamericana. 